\title{
The Impact of the Finite Element Method on Medical Device Design
}

\author{
Mark Driscoll ${ }^{1}$
}

Published online: 6 June 2018

(c) Taiwanese Society of Biomedical Engineering 2018

Over the last 10 years, medical device design has vastly benefited from the integration of finite element analyses in the product development process. The finite element method (FEM) is a numerical method used to solve boundary value problems. This method adopts an approach of computing reactions over a discrete number of points across the domain of interest. For medical design, this typically translates towards verifying device performance in a virtual domain that is representative of its planned real-life application. A user may leverage results from such an analysis to interpret device performance and make educated recommendations for improvement and optimization [1-3]. Moreover, predictions of initial and long-term device performance in vivo may also be derived from FEM results often through a multi scaled and iterative analyses $[4,5]$ which may subsequently lead to better prepared in vivo studies $[6,7]$. A key acknowledgement that enables such post-processing realizations is having an experienced user working with an interdisciplinary team. Such a team may then confidently navigate through the many advantages and disadvantages put forth by this virtual test platform which continues to grow in popularity.

The advantages of FEM in the medical device arena are many. The most attractive being the speed at which the FEM can enable early device performance testing prior to costly prototyping and bench testing. Correspondingly, integration of the FEM process into product development may reduce costs over the product development cycle. Such savings come to fruition by way of tentatively speeding up the process and reducing bench testing iterations. That is, bench testing may be performed via the FEM or in silico instead of on the bench side or ex vivo. The disadvantages of the FEM for medical device design reside mainly with the high expertise required to properly navigate the computational

Mark Driscoll

mark.driscoll@mcgill.ca

1 Department of Mechanical Engineering, McGill University, 817 Sherbrooke Street West, Macdonald Eng. Bldg. Office \#153, Montreal, QC H3A 0C3, Canada platform while avoiding making costly mistakes from ambitious misinterpretations. Further, established product development strategies must be revised to integrate the FEM into the early design phase which will take considerable effort for companies. Thus, the FEM has not completely shaken the perception of being a research tool rather than a development asset just yet.

One may become a skilled and experience FEM user through extensive knowledge of the mechanics behind the solver and awareness and implementation of verification and validation efforts. A specific and recommended guide now exist for verification and validation of numerical models in solid mechanics [8] and other fields. Verification is concerned with if the relations or equations are being solved correctly. Most commercial programs undergo stringent verification procedure prior to release. However, the functionality and proper convergence of the newly programed constitutive formulations used to represent the intricacies of a physiological environment of the medical device must be performed and reported. Furthermore, in addition to individual verification of such formulas, mechanical algorithms may give rise to new potential errors or instabilities when combined. Such compounded relations must also be verified and accounted for. Once the FEM appears to be verified, additional sensitivity or probabilistic analyses should be conducted to further weight the impact of your assumptions on the results [9]. If too much fluctuation in the results is initiated from the sensitivity study, the variable's magnitude must be selected carefully and results viewed and interpreted cautiously. If little fluctuations ensue then the formulation under consideration is robust. In contrast to verification, validation is concerned with if the correct relations or equations are being used. Thus, in other words, is one using the right mathematics that agree with the mechanics of the FEM solver. The most convincing form of validation is done experimentally by way of an experiment that is representative of the FEM simulated boundary value problem. This form of direct validation will either support or discredit what was reported utilizing the FEM platform. The user must however be aware of the statistical variances that 
occur with experimental practises and contrast the experimental and FEM platforms results accordingly. An indirect form of validation is achieved using literature data. Ideally, the literature data will be representative of the boundaries of the FEM platform which is often difficult to achieve. Most often, an array of literature data is adopted to gauge the validation of the FEM results. In this scenario, the term corroboration of results is more fitting than validation provided exact coherence is not possible. The above phases are merely a brief outline of what steps are required to extract clinically relevant information of FEM simulations [10]. One must also keep in mind the content of use for which the validation efforts were conducted. That is, the model is validated for that particular application or content of use and, should alternate simulations be desired, subsequent validation steps should ensue. Now, if an experienced user is able to verify and validate the FEM simulation and then leverage the FEM model to streamline or orient product development, how is this viewed by the regulatory bodies who are charged with the task of deciding upon the marketability or safety and effectiveness of medical devices?

The status of the FEM in the eyes of the regulatory bodies such as the Food and Drug Administration (FDA) in the United States of America or more specifically the branch of the Center for Devices and Radiological Health has taken some large steps towards FEM integration in product design dossiers. In particular, for many years industry has sought to leverage computational modeling such as FEM simulations towards support design safety and effectiveness in areas of fluid dynamics (ventricular devices) and solid mechanics (spine, knee, hip implants, etc.). In reaction, the FDA has drafted, in 2014, and later issued, in 2016, a guidance document entitled "Reporting of Computational Modeling Studies in Medical Device Submissions" [11]. Briefly, this public document provides informal guidelines on how to fully describe the modelling technique adopted and how it adheres to software quality assurance and numerical code verification expectations generically and then specifically to certain fields of application such as solid mechanics, for example. It would appear the justification for the worst-case scenario choices leading to subsequent bench testing may be acceptable but, to the author's experience in the solid mechanics field, FEM replacement of bench tests is not standard practice. An alternate hurdle exists in the internal regulatory team of the medical device companies who pose a reluctance, at least currently, to file regulatory dossiers that rely on FEM data which introduce uncertainty in a review process where delays are quite costly.

It is nevertheless apparent, with the growing adoption of FEM methods in medical device design and development, that regulatory bodies will adapt to these new trends. To this point, the FDA has been collaborating with many leading groups in this field on a V\&V40 ASME standard (Verification and validation in computational modeling of medical devices) document expected to released in 2018. This will elevate computational testing to equal consideration as bench, animal, and human testing currently receives-each testing method providing their specific advantages, disadvantages, requirements and expectations.

\section{References}

1. Driscoll, M., et al. (2013). Development of a detailed volumetric finite element model of the spine to simulate surgical correction of spinal deformities. BioMed Research International, 2013, 931741.

2. Driscoll, M., et al. (2013). Biomechanical assessment of reduction forces measured during scoliotic instrumentation using two different screw designs. Spine Deformity, 1(2), 94-101.

3. Driscoll, M., et al. (2015). Biomechanical comparison of 2 different pedicle screw systems during the surgical correction of adult spinal deformities. Spine Deformity, 3(2), 114-121.

4. Driscoll, M., et al. (2011). Biomechanical comparison of fusionless growth modulation corrective techniques in pediatric scoliosis. Medical \& Biological Engineering \& Computing, 49(12), 1437-1445.

5. Hachem, B., Aubin, C. E., Driscoll, M., \& Parent, S. (2012). Porcine spine finite element model as an alternative tool for the validation of a novel fusionless device. Studies in Health Technology and Informatics, 8(176), 459.

6. Driscoll, M., et al. (2016). Novel hemi-staple for the fusionless correction of pediatric scoliosis: Influence on intervertebral disks and growth plates in a porcine model. Clinical Spine Surgery, 29(9), 457-464.

7. Driscoll, M., et al. (2012). Spinal growth modulation using a novel intravertebral epiphyseal device in an immature porcine model. European Spine Journal, 21(1), 138-144.

8. ASME, A.S.o.M.E. (2006) Guide for verification and validation in computational solid mechanics.

9. Dar, F. H., Meakin, J. R., \& Aspden, R. M. (2002). Statistical methods in finite element analysis. Journal of Biomechanics, 35(9), 1155-1161.

10. Viceconti, M., et al. (2005). Extracting clinically relevant data from finite element simulations. Clinical Biomechanics, 20(5), 451-454.

11. FDA. (2016). Reporting of computational modeling studies in medical device submissions in Food and Drug Administration. Silver Spring, MD: Food and Drug Administration. 